\title{
The 46th annual meeting of the European Society for Blood and Marrow Transplantation: Van Bekkum awards
}

C Springer Nature Limited 2020

29 August -1 September, 2020 Virtual Meeting

Modified and published with permission from https://www.ebmt.org/annual-meeting

Sponsorship Statement: Publication of this supplement is sponsored by the European Society for Blood and Marrow Transplantation.

1998

Induction of alloantigen specific anergy does not impair cytolytic activity of leukaemia reactive human $\mathrm{T}$-cells

P. Comolie, F. Locatelli, D. Montagna, V. Calcaterra, A. Moretta, G. Giorgiani, F. Bonetti, M. Zecca, R. Maccario (Pavia, I)

\section{9}

First results of the autologous transplantation after serum-free retroviral gene marking of chemotherapy mobilised PBSC in chronic myeloid leukaemia

C. von Kalle, H. Glimm, S. Klingenberg, M. Schmidt, C. Waller, M. Engelhard, K. Flügge, G. Pache, C. Philbrook, T. Vraetz, J. Finke, R. Mertelsmann, W. Lange (Freiburg, D)

2000

Current results in haploidentical stem cell transplantation in children

T.E. Klingebiel, P. Lang, M. Schumm, P. Bader, P.G. Schlegel, M. Eyrich, J. Greil, D. Niethammer, R. Handgretinger (Tübingen, Erlangen, D)

2001

Generation of allo-HLA restricted minor histocompatibility antigen HA-1 specific cytotoxic T-cells (CTLs) as tools for treatment of relapsed leukemia following HLAmismatched stem cell transplantation

T. Mutis, E. Blokland, E. Schrama, E. Goulmy (Leiden, NL)

\section{2}

Striking survival advantage of bad-risk acute myeloid leukaemia patients transplanted from haploidentical donors with KIR epitope incompatibility in the GVH direction

L. Ruggeri, M. Capanni, E. Urbani, A. Tosti, K. Perruccio, S. Posati, F. Aversa, A. Tabilio, M.F. Martelli, A. Velardi (Perugia, I)

\section{3}

Differences in dendritic cell response to Aspergillus fumigatus as detected by gene expression profiling

H. Einsele, H. Hebart, C. Sinzger, S. Riegler, M. Bonin, J. Loeffler (Tübingen, D)

\section{4}

NOD2/CARD15 mutations of recipients and donors associate with GvHD and TRM following allogeneic stem cell transplantation-results in 2 independent cohorts of patients 
E. Holler, G. Rogler, J. Brenmoehl, A. Dickinson, G. Jackson, H. Greinix, G. Fischer, H. Herfarth, J. Hahn, G. Eissner, J. Schölmerich, R. Andreesen (Regensburg, D; Newcastle, UK; Vienna, A)

\section{5}

Clinical trials of immuno-gene therapy using tumour mRNA transfected dendritic cells

G. Gaudernack, S. Aamdal, S. Dueland, J. Kyte, L. Mu, S. Saeboe-Larssen, M. Hauser, H. Waehre, G. Kvalheim (Oslo, N)

\section{6}

Allogeneic SCT planned in CR-1 after intensive chemotherapy in patients with bad prognosis MDS results in better outcome in the intermediate/high-risk cytogenetic group: a study by the EORTC, EBMT, SAKK, HOVON and GIMEMA Leukemia Groups

T. de Witte, A. Hagemeijer, S. Suciu, A. Belhabri, M. Delforge, C. Aul, M. Aivado, D. Selleslag, H. Schouten, A. Ferrant, H. Biersack, S. Amadori, P. Muus, U. Jehn, F. Beeldens, J. Jansen, E. Hellstrom-Lindberg, T. Kovacsovics, U. Hess, P. Wijermans, G. Ossenkoppele, A. Gratwohl, J.-P. Marie, R. Willemze (Nijmegen, NL; Leuven, Brussels, B; Lyon, F; Duisburg, Düsseldorf, D; Brugge, B; Maastricht, NL; Essen, D; Rome, I; Munich, D; Stockholm, $\mathrm{S}$; Lausanne, St. Gallen, CH; The Hague, Amsterdam, NL; Basel, CH; Paris, F; Leiden, NL)

Mesenchymal stem cells for treatment of severe acute and extensive chronic graft-versus-host disease

O. Ringden, F. Frassoni, M. Uzunel, E. Lanino, B. Sundberg, H. Lonnies, G. Dini, A. Bacigalupo, F. Locatelli, W. Fibbe, K. Le Blanc (Stockholm, S; Genoa, I; Pavia, I; Leiden, NL)

2007

Single nucleotide polymorphisms of the NOD2/CARD15 gene are associated with significant increases in disease relapse and death in recipients of an unrelated donor haematopoietic stem cell transplant for acute leukaemia

N. Mayor, B. Shaw, D. Hughes, H. Maldonado-Torres, J.A. Madrigal, S. Keshav, S.G.E. Marsh (London, UK)
2008

Inhibitory KIR-ligand mismatching is associated with decreased incidence of relapse and improved diseasefree survival after unrelated cord blood stem cell transplantation for patients with acute leukaemia

R. Willemze*, C. Arrais Rodrigues, M. Labopin, I. Ionescu, K. Boudjedir, G. Sanz, G. Michel, G. Socié, B. Rio, J. Garcia, G. Kögler, L. Lecchi, P. Loiseau, E. Gluckman, V. Rocha on behalf of Eurocord-Netcord* and Acute Leukaemia Working Party of the EBMT, Paris

2009

Prophylactic transfer of Bcr/Abl, PR1 and WT1reactive donor $\mathbf{T}$-cells after $\mathbf{T}$-depleted allogeneic haematopoietic cell transplantation in patients with chronic myeloid leukaemia

M. Bornhäuser, C. Thiede, A. Kiani, U. Platzbecker, U. Oelschlägel, J. Radke, J. Schetelig, D. Lehmann, K. Hölig, G. Ehninger, M. Schmitz (Dresden, DE)

2010

Defibrotide prevents hepatic VOD and reduces significantly VOD-associated complications in children at high risk: final results of a prospective phase II/III multicentre study

S. Corbacioglu, S. Cesaro, M. Faraci, D. Valteau-Couanet, B. Gruhn, J. Boelens, C. Uderzo, N. Hewitt, A. Rovelli, J. Schrum, I. Müller, A. Schulz, J. Stein, R. Wynn, J. Greil, S. Matthes, K. Sykora, M. Albert, A. O'Meare, J. Massaro, R. D'Agostino, M. Pfitzenmaier, P. Sedlacek, P. Schlegel, J. Arvidson, J. Toporski, A. Groll, A. Fasth, J. Winiarski, H. Ozsahin, A. Schrauder, P. Bader, M. Hoyle, M. Iacobelli, G. Dini, C. Peters on behalf of the Pediatric Disease Working Party

\section{1}

Engineered T-lymphocytes using a novel anti-leukaemia antigen, aurora-A kinase-specific $\mathrm{T}$-cell receptor gene transfer successfully displayed anti-leukaemia reactivity

H. Fujiwara, K. Nagai, T. Ochi, J. An, T. Shirakata, J. Mineno, K. Kuzushima, H. Shiku, J. Melenhorst, E. Gostic, D. Price, M. Yasukawa (Toon, Ohtsu, Nagoya, Tsu, JP; Bethesda, US; Cardiff, UK) 
TCR gene editing results in effective immunotherapy of leukaemia without the development of GvHD

E. Provasi, P. Genovese, A. Lombardo, Z. Magnani, L. Pei-qi, A. Reik, V. Chu, D. Paschon, L. Zhang, J. Kuball, B. Camisa, A. Bondanza, G. Casorati, M. Ponzoni, F. Ciceri, C. Bordignon, P. Greenberg, M. Holmes, P. Gregory, L. Naldini, C. Bonini (Milan, IT, Richmond, Seattle, US)

\section{3}

Long-term outcomes after autologous haematopoietic cell transplantation for multiple sclerosis: a joint study from the Center for International Blood and Marrow Research (CIBMTR) and the European Group for Blood and Marrow Transplantat

P. Muraro* (London, Great Britain), M. Pasquini, H. Atkins, J. Bowen, D. Farge, A. Fassas, M. Freedman, G. Georges, N. Hamerschlak, E. Havdrova, T. Kozak, G. Luigi Mancardi, D. Morais, R. Nash, S. Pavletic, J. Ouyang, A. Saiz, M. Badoglio, X. Zhong, M. P. Sormani, R. Saccardi

\section{4}

T Cells Engineered With A Chimeric Antigen Receptor (Car) Targeting Cd19 (Ct1019 Cells) Produce Significant In Vivo Proliferation, Complete Responses And Longterm Persistence Without Gvhd In Children And Adults With Relapsed, Refractory All

S. Grupp* (Philadelphia, United States), N. Frey, R. Aplenc, B. Levine, S. Maude, S. Rheingold, C. Strait Barker, D. Teachey, Y. Mahnke, D. Porter, C. June

\section{5}

Improved cGvHD/relapse-free survival after HLAidentical sibling PBSC transplantation with ATG. A prospective multicenter randomized EBMT-labelled phase III trial (ATGfamilystudy)

C. Solano* (Valencia, Spain), F. Bonifazi, C. Wolschke, F. Patriarca, M. Pini, A. Nagler, M. Selleri, G. Messina, W. Bethge, P. Herrera Fuentes, R. Duarte, N. Cascavilla, M. Cimminiello, S. Guidi, J. Finke, A. Gallamini, C. Ferrà, J. Sierra, D. Russo, M. Petrini, G. Milone, F. Benedetti, M. Heinzelmann $^{3}$, V. Liso, M. Jurado, E. M. Pogliani, F. Narni, A. Völp, G. Bandini, F. Ayuk, T. Ruutu, N. Kröger on behalf of Chronic Malignancies Working Party of the EBMT (CMWP)
Multiple inhibitory receptors are expressed on central memory and memory stem $\mathbf{T}$ cells infiltrating the bone marrow of AML patients relapsing after allo-HSCT

Maddalena Noviello* (Milan, Italy), Francesco Manfredi, Tommaso Perini, Giacomo Oliveira, Filippo Cortesi, Cristina Toffalori, Valentina Gambacorta, Raffaella Greco, Jacopo Peccatori, Attilio Bondanza, Giulia Casorati, Paolo Dellabona, Nicoletta Cieri, Fabio Ciceri, Luca Vago, Chiara Bonini

\section{7}

Impact of MRD before and after Allogeneic Hematopoietic Cell Transplantation (HCT) of Childhood ALL By FC and RQ-PCR: A Retrospective Study on Behalf of COG, the PBMTC, the I-BFM the PDWP of the EBMT, and the Westhafen-Intercontinental-Group

Peter Bader* (Frankfurt, Germany), Emilia SalzmannManrique, Adriana Balduzzi, Jean-Hugues Dalle, Ann E. Woolfrey, Merav Bar, Michael R. Veneris, Michael J. Borowitz, Nirali Shah, Nathan Gossai, Peter Shaw, Allen Chen, Hermann Kreyenberg, Lucia Di Maio, Cornelia Eckert, Vincent van der Velden, Arjan Lankester, Thomas Klingebiel, Christina Peters, Stephan A. Grupp, Michael A. Pulsipher

\section{8}

Non-myeloablative haematopoietic stem cell transplantation versus continued disease modifying therapies (DMT) in patients with highly active relapsing remitting multiple sclerosis (RRMS)

Richard K Burt* (Chicago, USA), Roumen Balabanov, John A Snowden, Basil Sharrack, Maria Carolina Oliveira, Flavia Nelson, Joachim Burman

\section{9}

5-azacytidine (5-Aza) induction followed by allogeneic stem cell transplantation versus continuous 5 -Aza in elderly MDS patients (55-70 years). A prospective randomized study (VidazaAllo Study)

Nicolaus Kröger, Katja Sockel, Christine Wolschke, Wolfgang Bethge, Richard F. Schlenk, Dominik Wolf, Michael Stadler, Guido Kobbe, Gerald Wulf, Gesine Bug, Kerstin Schäfer-Eckart, Christoph Scheid, Florian Nolte, Jan Krönke, Matthias Stelljes, Dietrich Beelen, Marion 
Heinzelmann, Detlef Haase, Hannes Buchner, Gabriele Blecker, Uwe Platzbecker* (Frankfurt, Germany)

2020

Results of the EBMT SAAWP Phase III Prospective Randomized Multicenter RACE Study of Horse ATG and Ciclosporin with or without Eltrombopag in naïve SAA patients

Régis Peffault de Latour* (Paris, France), Judith Marsh, Simona Iacobelli, Sofie Terwel, Anita Hill, Constantijn J. M. Halkes, Christian Recher, Fiorenza Barraco, Edouard
Forcade, Juan Carlos Vallejo Llamas, Beatrice Dresler, Jean Baptiste Mear, Maria Teresa Van Lint, Reinier A.P. Raymakers, Marco R De Groot, Etienne Daguindau, Erfan Nur, Wilma Barcellini, Nigel H Russell, Louis Terriou, Anna Paola Iori, Isabel Sánchez- Ortega, Blanca Xicoy, Isidro Jarque, James Cavenagh, Flore Sicre de Fontbrune, Austin Kulasekararaj, Serena Marotta, Talha Munir, Jennifer M.L. Tjon, Suzanne Tavitian, Aline Praire, Laurence Clement, Florence Rabian, Alexander E Smith, Riley Cook, Luana Marano, Morag Griffin, Elena Palmisani, Petra Muus, Fabiana Cacace, Jakob R. Passweg, Gerard Socie, Ghulam J. Mufti, Carlo Dufour, Antonio Risitano 